\title{
Avaliação do uso do anel endocapsular modificado em casos de subluxação traumática do cristalino
}

\author{
Evaluation of the modified capsular tension ring in cases \\ of traumatic lens subluxation
}

\author{
Daniela Meira Villano Marques ${ }^{1}$ \\ Frederico França Marques $^{2}$ \\ Robert J. Cionni ${ }^{3}$ \\ Robert Henry Osher ${ }^{4}$ \\ Lincoln Lemes Freitas ${ }^{5}$
}

\section{RESUMO}

Objetivo: Avaliar o comportamento do anel modificado de tensão capsular em cataratas traumáticas com subluxação, analisando a acuidade visual, centração do saco capsular, pseudofacodonese, seguimento pósoperatório, e complicações intra e pós-operatórias. Local: Cincinnati Eye Institute, Cincinnati, Ohio, USA. Métodos: Vinte e dois olhos de 22 pacientes com perda traumática de suporte zonular foram submetidos à facoemulsificação utilizando lente intra-ocular de câmara posterior e o implante do anel capsular modificado. O exame pré-operatório incluiu melhor acuidade visual corrigida (MAVC), a presença ou ausência de facodonese, descentração do cristalino e prolapso vítreo. A avaliação pós-operatória incluiu MAVC, presença de pseudofacodonese, centração do implante, necessidade de vitrectomia e outras complicações. Resultados: Vinte e um olhos $(95,45 \%)$ tiveram melhora da MAVC. O exame pré-operatório revelou facodonese em 11 olhos (50\%) e nenhum olho apresentou pseudofacodonese. No exame pré-operatório, a descentração sintomática estava presente em 10 olhos $(45,45 \%)$. Todos os olhos apresentaram centração do complexo saco capsular/LIO no pós-operatório. Prolapso vítreo foi diagnosticado antes da cirurgia em 9 olhos (40,90\%) e a vitrectomia foi necessária em 11 olhos (50\%). Conclusão: $\mathrm{O}$ uso do anel modificado de tensão capsular resultou em boa centração do complexo saco capsular/lente intra-ocular nos olhos estudados com catarata traumática e perda de suporte zonular.

Descritores: Traumatismos oculares; Acuidade visual; Implante de lente intraocular; Extração de catarata

\section{INTRODUÇÃO}

Freqüentemente, os olhos com catarata traumática apresentam uma variedade de alterações morfológicas e funcionais dos segmentos anterior e posterior do olho ${ }^{(1-4)}$. Deve-se ressaltar que as lesões traumáticas do globo ocular constituem a principal causa de opacificação unilateral do cristalino e são consideradas a principal causa de cegueira em adulto jovem ${ }^{(5-6)}$.

O exame pré-operatório de biomicroscopia do segmento anterior deve ser realizado cuidadosamente buscando avaliar a condição exata do cristalino, pois não é sempre que nos deparamos com achados clínicos de subluxação (facodonese, iridodonese, vítreo em câmara anterior), sendo assim, os sinais discretos de subluxação devem ser pesquisados (iridodonese focal, espaço iridolenticular) visando um planejamento cirúrgico mais preciso ${ }^{(7)}$.

O anel endocapsular (CTR) foi idealizado em 1995 para casos de deficiência zonular progressiva e grave ${ }^{(8-9)}$. Em 1998, Cionni e Osher introduzi- 
ram o uso do anel endocapsular modificado (MCTR, Morcher $\mathrm{GmbH})$. Este incorporou ao anel endocapsular original (CTR) um gancho que possibilita a fixação na esclera sem violar a integridade do saco capsular, podendo ser utilizado para mais de 120 graus de lesão zonular.

Várias vantagens do anel endocapsular têm sido descritas na cirurgia de catarata $^{(8,10-12)}$. Dentre elas, temos: 1- estabilizar a cápsula e aumentar a segurança e eficácia durante a facoemulsificação e implante de lente intra-ocular (LIO) de câmara posterior; 2- manter o contorno circular do saco capsular e evitar o seu colabamento uma vez que o cristalino foi removido; 3- proporcionar suporte adicional ao saco capsular pois empurra o equador do cristalino para a posição original, o que ajuda a impedir a descentração e contração em casos de zônula frágil e fibrose capsular; 4- inibir a migração de células epiteliais para a cápsula posterior por compressão física e barreira funcional, o que reduz a formação de catarata secundária especialmente se o anel apresentar borda vertical ${ }^{(12)}$.

Este estudo tem como objetivo estudar o comportamento do anel endocapsular modificado em casos de subluxação traumática do cristalino por meio da avaliação da acuidade visual, centração do saco capsular, pseudofacodonese, acompanhamento pós-operatório e complicações intra e pós-operatórias.

\section{MÉTODOS}

Foram estudados de forma retrospectiva 22 olhos de 22 pacientes com catarata traumática do Cincinnati Eye Institute (CEI) no período de novembro de 1997 a julho de 2001 operados pelo mesmo cirurgião, utilizando a mesma LIO e o mesmo tipo de anel (MCTR-L1).

A composição da amostra estudada foi de 12 (54,54\%) homens para $10(45,45 \%)$ mulheres. A idade dos pacientes variou de 3 a 80 anos, sendo a média 48,8 anos.

A cirurgia de catarata foi indicada quando o paciente apresentou catarata com baixa de acuidade visual secundária ao trauma e subluxação de cristalino. $\mathrm{O}$ intervalo entre o trauma ocular e a cirurgia variou entre 0,25 meses e 372 meses.

$\mathrm{O}$ exame pré-operatório era composto por anamnese e exame oftalmológico completo. Para nossa avaliação, consideramos a melhor acuidade visual corrigida (MAVC), análise da presença ou ausência de facodonese, descentração do cristalino e prolapso vítreo para câmara anterior pelo exame de biomicroscopia.

Os pacientes foram submetidos ao procedimento de facoemulsificação com implante de lente intra-ocular de câmara posterior (PC-LIO) e do anel endocapsular modificado tipo 1L (MCTR- Modified Capsular Tension Ring). Os pacientes foram acompanhados no pós-operatório durante um período que variou entre 4 a 38 meses (média de 15,81 meses).

\section{Procedimento cirúrgico}

A incisão deve ser realizada longe da área da diálise zonular, pois irá ajudar a reduzir o estresse na zônula durante a facoemulsificação.
A entrada cautelosa na câmara anterior deve ser do tamanho suficiente para a entrada da cânula do viscoelástico. Coloca-se o material viscoelástico sobre a área da diálise, com intuito de tamponar o vítreo e manter a profundidade da câmara anterior.

Deve-se iniciar a capsulorrexis em uma área longe da diálise. O seu tamanho final deve ser em torno de 5,5 a 6,0 mm.

A hidrodissecção deve ser realizada cuidadosamente. Em casos de núcleos moles, é aconselhável que se faça uma hidrodissecção completa, luxando o núcleo para a câmara anterior.

A facoemulsificação deve ser realizada utilizando baixos parâmetros de vácuo e aspiração e a altura do frasco também deve permanecer baixa ${ }^{(13)}$. A técnica de faco/chop é a de escolha para casos com fragilidade zonular, pois diminui o estresse na zônula.

A aspiração do material cortical é preferencialmente manual, com uma cânula de 24 a 27 gauge, de forma tangencial ao saco capsular. Caso apareça vítreo durante o procedimento, ele deve ser completamente removido da câmara anterior pela técnica de vitrectomia sem infusão, a seco, e a câmara anterior preenchida por viscoelástico se a quantidade de vítreo for pequena. Caso o prolapso vítreo seja volumoso, a vitrectomia bimanual é indicada.

Para a colocação do anel, inicialmente passa-se o fio de sutura prolene 9.0 no gancho do anel. Este então é inserido dentro do saco capsular e o fio é passado através da parede da esclera sem violar a integridade da cápsula anterior compondo uma sutura em "U” (Figura 1 - A, B, C e D). Estes pontos são cobertos por um "flap" escleral.

A colocação da LIO dentro do saco capsular é muito mais difícil em casos de diálise zonular, portanto, em todos os casos deste estudo o anel endocapsular modificado foi colocado antes da inserção da lente.

Após o saco capsular estável, ele é preenchido com viscoelástico para a LIO ser inserida através do injetor. Removese o material viscoelástico com aspiração mecanizada, injetase miostático intracameral e termina-se o procedimento com injeção de solução balanceada no estroma da incisão corneana a fim de torná-la autoselante.

Em nenhuma das cirurgias realizadas houve necessidade de curativo oclusivo.

No período pós-operatório, observamos a MAVC, presença ou ausência de pseudofacodonese e descentração do saco capsular.

Para avaliarmos possível diferença de comportamento do pré-operatório para o pós-operatório em relação às variáveis previamente definidas, usamos para a análise estatística o teste dos sinais (Siegel) e foi considerado estatísticamente significativo quando o valor $\mathrm{p}<0,005^{(14)}$.

\section{RESULTADOS}

\section{Melhor acuidade visual corrigida}

De acordo com a análise da acuidade visual pré-operatória, 11 olhos $(50,00 \%)$ apresentaram MAVC menor ou igual a 


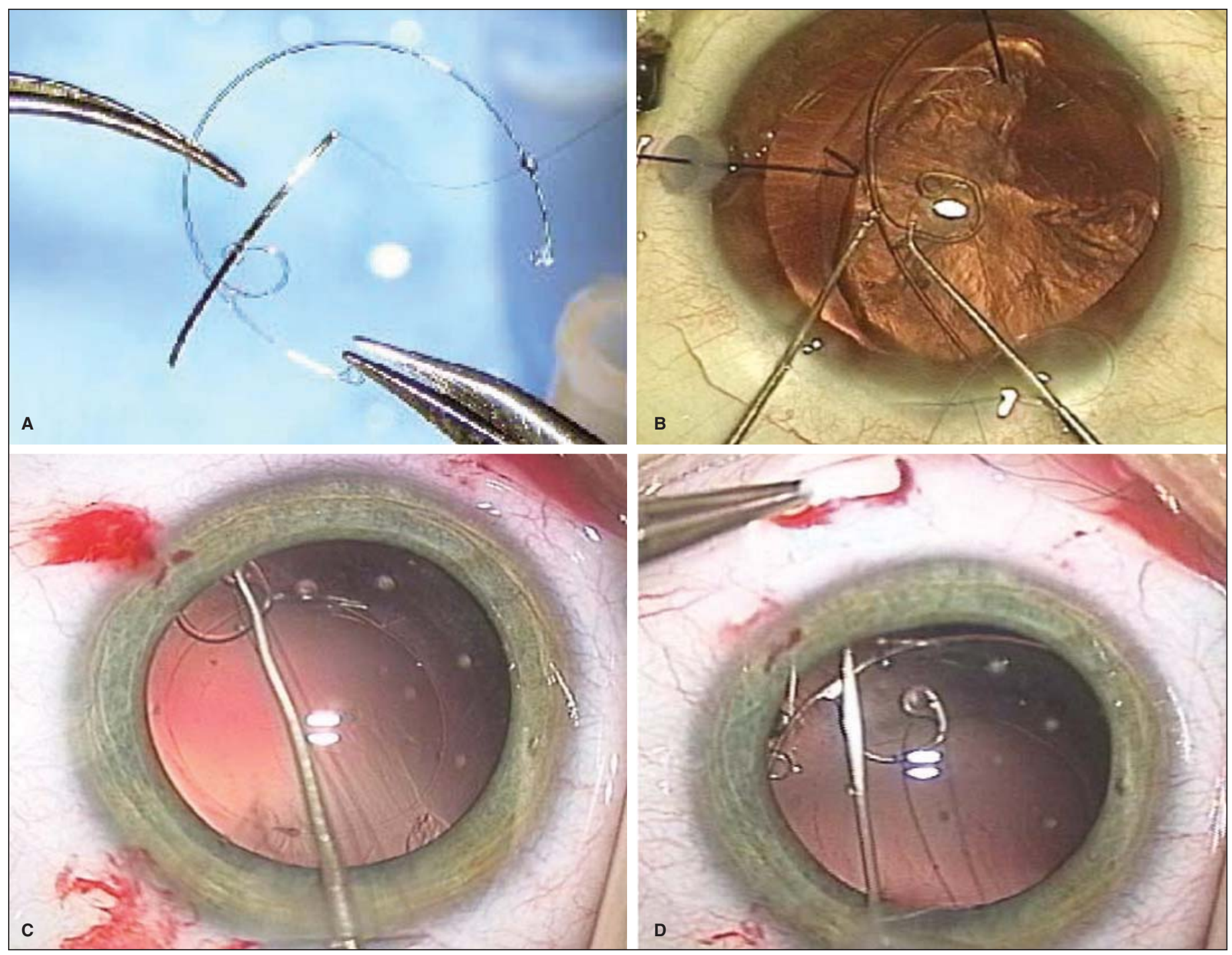

Figura 1- A) Fio de sutura passado através do orificio de fixação do anel; B) Inserção do anel no saco capsular; C) Posicionamento final do anel;

D) Sutura do anel na parede escleral

20/200, 8 olhos $(36,36 \%)$ apresentaram MAVC entre 20/40 e 20/200 e 3 olhos (13,63\%) apresentaram MAVC maior ou igual a 20/40. Ao último exame pós-operatório, 21 olhos $(95,45 \%)$ mostraram melhora da MAVC, 1 olho $(4,55 \%)$ manteve a MAVC e nenhum dos olhos apresentou redução de linhas na tabela de acuidade visual de Snellen. De acordo com a análise pós-operatória, 2 olhos (9,09\%) apresentaram MAVC entre 20/200 e 20/40, sendo em olhos que apresentavam previamente glaucoma e cicatriz macular, e 20 olhos $(90,90 \%)$ apresentaram MAVC maior ou igual 20/40, como mostra a tabela 1 .

\section{Facodonese e pseudofacodonese}

A estabilidade do cristalino foi avaliada durante o exame pré-operatório e revelou que 11 olhos (50\%) apresentavam facodonese. No entanto, no exame pós-operatório, em nenhum olho foi observado pseudofacodonese, mostrando uma imporatante estabilidade do complexo saco capsular/LIO após a cirurgia, como mostra a tabela 2 .

\section{Descentração}

A extensão da descentração do saco capsular não foi medida na avaliação pré-operatória e na pós-operatória. $\mathrm{O}$ cristalino e a LIO foram considerados descentrados em situações em que o paciente referiu visão dupla ou imagens ópticas indesejadas por consequência do cristalino ou da LIO (descentração sintomática).

Descentração sintomática estava presente em 10 olhos $(45,45 \%)$ antes da cirurgia. Os 22 olhos (100\%) tiveram suas LIOs centradas após a cirurgia e mantiveram as LIOs centradas até o final do acompanhamento, como mostra a tabela 3.

\section{Diálise zonular}

A extensão da diálise zonular foi estudada e caracterizada 
no intra-operatório, apresentando uma variação de 60 a 320 graus, cuja média foi de 187,27 graus o desvio padrão foi de $\pm 57,17$ graus.

Pode-se notar que, mesmo em casos cuja diálise zonular foi mais intensa, ou seja, maior do que 120 graus, obteve-se uma melhora importante da descentração após a cirurgia.

\section{Prolapso vítreo e vitrectomia}

No exame pré-operatório, prolapso vítreo foi encontrado na câmara anterior em 9 olhos (40,90\%), enquanto que o procedimento vitrectomia foi necessário em 11 olhos (50\%), conforme mostra a tabela 4 .

\section{Complicações pós-operatórias}

As complicações encontradas no pós-operatório foram:

1) aumento transitório da PIO em 4 olhos (18,18\%), que resolveram com uso de medicação tópica, apareceu no primeiro mês pós-operatório;

2) aumento permanente da PIO em 1 olho (4,54\%), do primeiro dia pós-operatório até o final do acompanhamento;

3) opacidade da cápsula posterior em 9 olhos (40,90\%), apareceram a partir de 22 semanas até 28 meses de pósoperatório;

4) necessidade do uso do YAG laser em 7 olhos (31,81\%);

5) contração capsular em 1 olho (4,54\%) com 2 meses de pós-operatório;

6) descolamento de retina necessitando de vitrectomia posterior com "peeling" de membrana e "buckle" escleral e troca fluído-gasosa em 1 olho (4,54\%), aparecendo com 2 meses de pós-operatório.

As complicações que mais apareceram conjuntamente foram aumento transitório da PIO e opacificação da cápsula posterior em 4 casos $(18,18 \%)$. No entanto, os olhos que necessitaram fazer YAG devido à opacidade da cápsula posterior não apresentaram complicações secundárias a este procedimento.

\begin{tabular}{|lcc|}
\hline \multicolumn{3}{|c|}{ Tabela 1. Acuidade visual } \\
\cline { 2 - 3 } MAVC & \multicolumn{2}{c|}{ Número de olhos (\%) } \\
\cline { 2 - 3 }$\geq 20 / 40$ & $3(13,63 \%)$ & $20(90,90 \%)$ \\
$>20 / 200$ e $<20 / 40$ & $8(36,36 \%)$ & $2(9,09 \%)$ \\
$\leq 20 / 200$ & $11(50,00 \%)$ & $0(0 \%)$ \\
MAVC= Melhor acuidade visual corrigida medida na tabela de Snellen \\
Teste do Sinal; $N=22 ; X=1 ; p<0,000$ \\
\hline
\end{tabular}

Tabela 2. Presença de facodonese e pseudofacodonese

\begin{tabular}{lcc|} 
& \multicolumn{2}{c|}{ Número de olhos (\%) } \\
\cline { 2 - 3 } Facodonese & Pré-operatório & Pós-operatório* \\
Sim & $11(50 \%)$ & $0(0 \%)$ \\
Não & $11(50 \%)$ & $22(100 \%)$ \\
${ }^{*}$ Pseudofacodonese & & \\
$\mathrm{N}=22, \mathrm{x}=0, \mathrm{p}<0,000 ; \mathrm{N}=11 \mathrm{x}=0, \mathrm{p}<0,000$ & \\
\hline
\end{tabular}

\section{DISCUSSÃO}

A adequada centralização da LIO e a estabilidade do saco capsular são críticos para a manutenção de uma boa acuidade visual final. Fato comprovado em nosso estudo pela melhora da acuidade visual final em todos os casos $(95,45 \%)$, com exceção de um único olho (4,55\%) que teve sua acuidade visual final inalterada.

Podemos avaliar o comportamento do MCTR em um estudo de 90 olhos, com comprometimento zonular congênito. Houve melhora da acuidade visual final em 79 olhos $(87,8 \%), 1$ olho $(1,1 \%)$ apresentou piora e em 9 olhos $(10 \%)$ não houve alteração da acuidade visual ${ }^{(15)}$.

Com a presença do anel, a barreira anatômica cápsulazônula é parcialmente reformada, então o prolapso vítreo na câmara anterior durante a cirurgia é reduzido ou prevenido ${ }^{(9,16)}$. Assim como observamos em nosso estudo, 9 olhos (40,90\%) apresentaram prolapso vítreo no pré-operatório e 11 olhos $(50 \%)$ necessitaram ser submetidos à vitrectomia durante a cirurgia. Enquanto, no estudo similar, prolapso vítero estava presente no pré-operatório em 11 olhos $(12,2 \%)$ e 14 olhos $(15,6 \%)$ necessitaram de vitrectomia durante a cirurgia ${ }^{(15)}$.

Apesar de se constituírem em cirurgias difíceis e haver riscos em potencial, existem poucos relatos referentes às complicações tardias ou precoces ${ }^{(17-18)}$. Em nosso estudo, foram encontradas as seguintes complicações tardias e precoces: aumento transitório $(18,18 \%)$ e permanente $(4,54 \%)$ da PIO, OCP (40,9\%), contração capsular (4,54\%) e descolamento de retina $(4,54 \%)$. Enquanto, que no estudo similar as seguintes complicações ocorreram: aumento transitório da PIO (2,2\%), descolamento de retina (1\%), irite persistente $(3,3 \%)$, quebra do fio de sutura $(10 \%)$ o que resultou na troca do Prolene 10-0 pelo 9-0 e OCP $(22 \%)^{(15)}$.

Um caso de fimose capsular em olho com anel endocapsular e LIO de PMMA, mostrou que em casos de lesão zonular, a fixação escleral pela "eyelet" com prolene 9-0, apesar de tecnicamente mais dificil, parece ser a melhor opção ${ }^{(19)}$. Em nosso estudo apenas 1 olho (4,54\%) apresentou contração capsular.

\begin{tabular}{|lcc|}
\hline \multicolumn{3}{|c|}{ Tabela 3. Descentração pré e pós-operatória } \\
\cline { 2 - 3 } Descentração & Número de olhos (\%) \\
\cline { 2 - 3 } Sim & $10(45,45 \%)$ & $0(0 \%)$ \\
Não & $12(54,54 \%)$ & $22(100 \%)$ \\
$N=22 x=0, p=0,000 ; N=10 x=0, p=0,001$ ou $0,1 \%$ & \\
\hline
\end{tabular}

\begin{tabular}{|lcc|}
\hline \multicolumn{3}{|c|}{ Tabela 4. Prolapso vítreo x vitrectomia } \\
\cline { 2 - 3 } & \multicolumn{2}{c|}{ Prolapso vítreo } \\
\cline { 2 - 3 } Vitrectomia & Número de olhos (\%) & Número de olhos (\%) \\
Sim & $5(22,72 \%)$ & $6(27,27 \%)$ \\
Não & $4(18,18 \%)$ & $7(31,81 \%)$ \\
\hline
\end{tabular}


Em nosso estudo, observamos que antes da cirurgia, 10 olhos $(45,45 \%)$ apresentavam descentração do cristalino, enquanto que no pós-operatório nenhum olho apresentou descentração das LIOs, embora haja estudo com relatos de descentração pós-operatória leve, porém, sem sintomas aos pacientes os quais se apresentavam satisfeitos ${ }^{(20)}$.

No estudo similar, 86 olhos $(95,6 \%)$ apresentavam descentração do cristalino no pré-operatório, enquanto que 84 destes olhos mantiveram-se com boa centração no pós-operatório. Os 6 olhos $(6,7 \%)$ restantes desenvolveram descentração tardia pela quebra do fio de sutura, precisando ser submetidos à ressutura ${ }^{(15)}$.

É muito difícil realizar uma CCC bem centrada em olhos com subluxação do cristalino. Este fato propicia a formação de OCP periférica e depois central(21). Caso seja necessário realizar capsulotomia com Nd:YAG laser, este deve ser feito com cautela para que não cause descentração da $\mathrm{LIO}^{(20)}$.

Em nosso estudo, observamos que em 9 olhos (40,90\%) houve a formação de OCP a partir de 22 semanas até 28 meses, sendo que em 7 olhos $(31,81 \%)$ foi necessário realizar a capsulotomia com Nd:YAG laser. No entanto, não constatamos nenhuma complicação relacionada ao procedimento mencionado. Explicamos este número elevado pelo fato de que a aspiração do material cortical residual não é realizado vigorosamente como em casos convencionais, pelo fato do saco capsular apresentar-se mais frágil.

Complicações aumentam em pacientes com fragilidade zonular. Grande parte $(60 \%)$ dos pacientes com fragilidade zonular confirmados com facodonese desenvolve perda vítrea durante a cirurgia, segundo a literatura $^{(22)}$. A taxa de complicações foi maior nos dois grupos quando comparada ao de cirurgias de catarata senil ${ }^{(23)}$. Em nosso estudo, 4 olhos $(18,18 \%)$ apresentaram perda vítrea durante a cirurgia.

A PIO aumentou freqüentemente, porém não houve casos de glaucoma secundário à cirurgia de catarata. Em nosso estudo, apenas 1 olho (4,54\%) desenvolveu aumento permanente da PIO, sendo que era um olho previamente glaucomatoso, enquanto 4 olhos $(18,18 \%)$ desenvolveram aumento transitório da PIO, que posteriormante resolveram com medicação tópica.

\section{CONCLUSÃO}

O anel endocapsular modificado (MCTR) apresenta um comportamento positivo e estável em situações cirúrgicas de extrema dificuldade como a proposta neste estudo (catarata traumática). Possibilitando assim, a manutenção de uma acuidade visual satisfatória pós-operatória, centralização da LIO pós-operatória, ausência de pseudofacodonese e com poucas complicações intra e pós-operatórias.

\section{ABSTRACT}

Purpose: To evaluate the behavior of the modified capsular tension ring (MCTR) in traumatic subluxated cataracts analyzing the visual acuity, centration of the capsular bag, pseudophacodonesis, postoperative follow-up and intra- and postoperative complications. Setting: Cincinnati Eye Institute, Cincinnati, Ohio, USA. Methods: Twenty-two eyes of 22 patients with traumatic loss of zonular support had phacoemulsification using PCIOL and MCTR implantation. The preoperative examination included best correct visual acuity (BCVA) and the presence or absence of phacodonesis, lens decentration and vitreous prolapse. The postoperative evaluation included BCVA, the presence of pseudophacodonesis, PCIOL centration, necessity of vitrectomy and other complications. Results: 21 eyes $(95.45 \%)$ had an improvement in BCVA. The preoperative examination disclosed phacodonesis in 11 eyes (50\%) and no eye presented pseudophacodonesis in the postoperative period. Preoperatively, symptomatic decentration was presented by 10 eyes $(45.45 \%)$ and 22 eyes $(100 \%)$ had no decentration after surgery. The preoperative examination revealed vitreous prolapse in the anterior chamber in 9 eyes (40.90\%). Vitrectomy was required in 11 eyes (50\%). Conclusions: The use of the MCTR resulted in a good centration of the capsular bag and the PC IOL in 22 eyes with traumatic cataract and loss of zonular support.

Keywords: Eye injuries; Visual acuity; Lens implantation, intraocular; Cataract extraction

\section{REFERÊNCIAS}

1. Dannenberg AL, Parver LM, Brechner RJ, Khoo L. Penetration eye injuries in the workplace. The National Eye Trauma System Registry. Arch Ophthalmol. 1992;110(6):843-8.

2. Kazokoglu H, Saatci O. Intraocular foreign bodies: results of 27 cases. Ann Ophthalmol. 1990;22(10):373-6.

3. Koster HR, Kenyon KR. Complications of surgery associated with ocular trauma. Int Ophthalmol Clin.1992;32(4):157-78.

4. Blum M, Tetz MR, Greiner C, Voelcker HE. Treatment of traumatic cataracts. J Cataract Refract Surg. 1996;22(3):342-6.

5. Luntz MH. Clinical types of cataract. In: Tasman W, Jaeger EA, editors. Duane's clinical ophthalmology. Philadelphia: Harper \& Row; 1981. v.1. p.1-19. No texto.

6. Parver LM. Eye trauma. The neglected disorder. Arch Ophthalmol. 1986;104 (10):1452-3

7. Marques DM, Marques FF, Osher RH. Sutle signs of zonular damage. J Cataract Refract Surg. 2004;30(6):1295-9.

8. Cionni RJ, Osher RH. Endocapsular ring approach to the subluxed cataractous lens. J Cataract Refract Surg. 1995;21(3):245-9. Comment in: J Cataract Refract Surg. 2001;27(11):1710-1.

9. Hara T, Hara T, Sakanishi K, Yamada Y. Efficacy of equator rings in an experimental rabbit study. Arch Ophthalmol. 1995;113(8):1060-5.

10. Gimbel HV, Sun R, Heston JP. Management of zonular dialysis in phacoemulsification and IOL implantation using the capsular tension ring. Ophthalmic Surg Lasers. 1997;28(4):273-81.

11. Sun R, Gimbel HV. In vitro evaluation of the efficacy of the capsular tension ring for managing zonular dialysis in cataract surgery. Ophthalmic Surg Lasers. 1998;29(6):502-5.

12. Nishi O, Nishi K, Menapace R. Capsule-bending ring for the prevention of capsular opacification: a preliminary report. Ophthalmic Surg Lasers. 1998;29 (9):749-53.

13. Osher RH, Marques FF, Marques DM, Osher JM. Slow-motion phacoemulsification technique. Tech Ophthalmol. 2003;1(2):73-9.

14. Siegel S. Estatística não-paramétrica para as ciências do comportamento. São Paulo: McGraw-Hill do Brasil; 1975. p.350.

15. Cionni RJ, Osher RH, Marques DM, Marques FF, Snyder ME, Shapiro S. 
Modified capsular tension ring for patients with congenital loss of zonular support. J Cataract Refract Surg. 2003;29(9):1668-73.

16. Jacob S, Agarwal A, Agarwal A, Agarwal S, Patel N, Lal V. Efficacy of a capsular tension ring for phacoemulsification in eyes with zonular dialysis. $\mathrm{J}$ Cataract Refract Surg. 2003;29(2):315-21.

17. Dietlein TS, Jacobi PC, Konen W, Krieglstein GK. Complications of endocapsular tension ring implantation in a child with Marfan's syndrome. J Cataract Surg. 2000;26(6):937-40.

18. Faschinger CW, Eckhardt M. Complete capsulorhexis opening occlusion despite capsular tension ring implantation. J Cataract Refract Surg. 1999;25(7): 1013-5. Comment in: J Cataract Refract Surg. 2001;27(3):342-3.

19. Waheed K, Eleftheriadis H, Liu C. Anterior capsular phimosis in eyes with a capsular tension ring. J Cataract Refract Surg. 2001;27(10):1688-90.
20. Kohnen T, Baumeister M, Bühren J. Scheimpflug imaging of bilateral foldable in-the-bag intraocular lens implantation assisted by a scleral-sutured capsular tension ring in Marfan's syndrome. J Cataract Refract Surg. 2003;29 (3):598-602.

21. Hollick EJ, Spalton DJ, Meacock WR. The effect of capsulorhexis size on posterior capsular opacification: one-year results of a randomized prospective trial. Am J Ophthalmol. 1999;128(3):271-9.

22. Shingleton BJ, Heltzer J, O’Donoghue MW. Outcomes of phacoemulsification in patients with and without pseudoexfoliation syndrome. J Cataract Refract Surg. 2003;29(6):1080-6. Comment in: J Cataract Refract Surg. 2004;30(4):733; author reply 733.

23. Tetz M, Blum M, Greiner C, Völcker HE. Traumatische Katarakte. Operationsmethoden und Ergebnisse bei 106 Patienten. Ophthalmologe. 1993;90(4):360-3. 